Abstract IDDF2019-ABS-0022 Table 1 Change in clinical scoring system and clinical outcome from baseline to day 3

\begin{tabular}{|l|l|l|l|}
\hline & \multicolumn{1}{|c|}{ Pentoxifylline(n=26) } & \multicolumn{1}{|c|}{ Control( $n=24)$} & p-value \\
\hline Clinical Scoring system* & \multicolumn{1}{|c|}{} & \\
\hline APACHE II score & $0(-5$ to 14$)$ & $-2(-7$ to 14$)$ & 0.27 \\
\hline BISAP & $0(-2$ to 2$)$ & $0(-2$ to 1$)$ & 0.91 \\
\hline SIRs & $0(-3$ to 1$)$ & $0(-3$ to 1$)$ & 0.82 \\
\hline $\begin{array}{l}\text { Presence of SIRs at day 3, } \\
n(\%) * *\end{array}$ & $2(7.7 \%)$ & $7(29.2 \%)$ & 0.048 \\
\hline $\begin{array}{l}\text { Percentage change in } \\
\text { APACHE II score }\end{array}$ & $0(-100$ to 500$)$ & $-32(-100$ to 233.3$)$ & 0.3 \\
\hline
\end{tabular}

through TNF-a inhibition. However, its benefit in acute pancreatitis in human remains unclear.

Subjects To study clinical outcomes of pentoxifylline in APACHE II score in acute pancreatitis patients at 72 hours after treatment and to study the effects of pentoxifylline on inflammatory markers level.

Methods 54 acute pancreatitis patients with associated risk factors of severe pancreatitis development were evaluated for the severity of disease and inflammatory markers prior to treatment. Participants were allocated within 48 hours of diagnosis into pentoxifylline or control arm. The severity of disease, as well as inflammatory markers, were re-evaluated at 72 hours after treatment.

Results Pentoxifylline did not decrease the severity of disease determined by a reduction in APACHE II score and a percent reduction of APACHE II compared with control group (0 Vs. 2 ; p-value $=0.27$ and $0 \%$ reduction vs. $32 \%$ reduction; pvalue $=0.3$, respectively). Interestingly, the incidence of the systemic inflammatory responds syndrome (SIRS) after 72 hours of treatment was significantly lower than those without pentoxifylline. $(7.7 \%$ Vs. $29.2 \%$; p-value $=0.048)$ (table 1.). Noticeably in subgroup analysis, patients who enrollment time less than 24 hours after onset of symptoms show mean proinflammatory marker tended to respond to pentoxifylline group better than the control group.

Conclusions Pentoxifylline seems to reduce the inflammatory process of the early phase of acute pancreatitis, particularly in patients presented within 24 hours of onset. However, the overall severity of the disease and clinical benefit was similar to the control group.

\section{IDDF2019-ABS-0025 DEMOGRAPHIC PROFILE AND CLINICAL PRESENTATION OF IBD AMONG INPATIENTS SEEN FROM 2012-2018: A UERMMMC EXPERIENCE}

Millette Castro*. University of the East Ramon Magsaysay Memorial Medical Center, Philippines

\subsection{6/gutjnl-2019-IDDFabstracts.120}

Significance In the past, there has been a paucity of clinical and demographic information on inflammatory bowel disease in the Philippines. At the UERMMMMC, there was a growing trend for IBD diagnosis for the last $\underline{6}$ years.
Objective To provide clinical and demographic data of IBD among patients seen in our hospital.

Methods Review of histopathology logbook and endoscopy reports of diagnosed cases of inflammatory bowel disease at the UERMMMC from 2012-2018.

Results A total of 24 patients with a diagnosis of IBD were included. 15 patients have Crohn's disease (62.5\%) and 9 has Ulcerative Colitis (37.5\%). An average of 2-3 patients was diagnosed every year with 5-6 new cases diagnosed over the last $\underline{2}$ years. The mean annual incidence was $\underline{3}$ new cases with more males than females (19 vs. 5). Mean age was 40 years old with bimodal peak in the age at presentation among 21-40 years old and 40-60 years old. Common symptoms included diarrhea (58.3\%), abdominal pain $(20.8 \%)$, lower GI bleeding (12.5\%) and recurrent perianal fistula $(8.3 \%)$. 2 cases were negative at the onset. 2 cases had MTB co-infection and 1 case with HIV coinfection but had a biopsy suggestive only for Crohn's. 1 case had esophageal fistula. Of the UC cases, 90\% had rectosigmoid colitis and 10\% had pancolitis. Among CD, $60 \%$ had pure colitis, $35 \%$ had ileo-colitis and $5 \%$ had terminal ileitis.

Recommendations IBD is an emerging disease. More males were affected with histology consistent with Crohn's disease. Diarrhea remains to be the predominant symptom and it is very important to identify if there has been any co-existing infection like HIV and TB as this will greatly impact the treatment.

\section{IDDF2019-ABS-0031 REAL WORLD ANALYSIS ON THE DIAGNOSIS, TREATMENT PATTERNS AND OUTCOMES FOR PATIENTS WITH GASTRO- ESOPHAGEAL REFLUX DISEASE IN CHINA (GRAND CHINA STUDY): DESIGN AND METHOD}

${ }^{1}$ Xiaohua Hou* ${ }^{1}$ Xiaoyun Yu, ${ }^{2}$ Li Xie. ${ }^{1}$ Division of Gastroenterology, Union Hospital, Tongji Medical College, Huazhong University of Science and Technology, Wuhan, China; ${ }^{2}$ Medical affairs, Takeda china, China

\subsection{6/gutjnl-2019-IDDFabstracts. 121}

Background Gastro-oesophageal reflux disease (GERD) is a long-term condition where stomach contents come back into the esophagus resulting in either symptoms or complications. Real-world studies on GERD mainly focus on the 
prevalence as well as their associated patient characteristics and effective treatment rates. Few studies investigating treatment patterns of GERD have been conducted. Thus, it is significant to investigate current diagnostic practices and treatment patterns of GERD in China and understand the effectiveness of current standard treatments in real-world settings.

Methods The overall analysis population will consist of patients of all ages who were diagnosed with GERD from January 2015 to December 2017. Only the patient's first treatment trajectory will be counted during this period. Specific analysis population details for diagnosis, treatment, and real-world (RW) treatment efficacy are also outlined separately.

Results The sample size is currently unknown but will be determined based on three years of available GERD patient data within target hospitals' scope. The project sample will be selected across 20 hospitals in China. Data from 20 hospitals will be extracted from hospital electronic system. Variables of interest will cover patient socio-demographic characteristics (age, sex, health plan type, hospital, etc.), diagnostic methods, selected treatment details, selected medical history and medication use prior to and after the index date, and medical records of healing over the analysis period. The primary objective is to investigate current diagnostic practices and treatment patterns of GERD in China, including (1) Proportion and trend of GERD patients in different specialist outpatient clinics (digestive, respiratory, otolaryngology, others). (2) Treatment pattern (the treatment of different specialists, treatment of different types of symptoms, the relationship between symptoms and different endoscopic manifestations) of GERD in clinical practice.

Conclusions This GRAND CHINA STUDY would provide important evidence for current diagnostic practices and treatment patterns of GERD as well as evaluate the real-world outcomes of EE in China.

\section{IDDF2019-ABS-0035 THE EFFICACY OF COMBINING SIMETHICONE AND POLYETHYLENE GLYCOL FOR ADENOMA DETECTION DURING COLONOSCOPY: A SYSTEMATIC REVIEW AND META-ANALYSIS OF RANDOMIZED CONTROLLED TRIALS}

${ }^{1} \mathrm{Hu}$ Zhang*, 'Song-Lin Ma, ${ }^{1}$ Jing Liu, ${ }^{1}$ Man-Lin Huang, ${ }^{1}$ Qi-Long Song, ${ }^{1}$ Sheng-Bin Sun, ${ }^{1}$ Yan Fan, ${ }^{1}$ Min Song, ${ }^{1}$ Heng Zhang, ${ }^{2}$ Hui-Hua Luo, ${ }^{2}$ Chang Zhao. ${ }^{1}$ The Central Hospital of Wuhan, Tongji Medical College, Huazhong University of Science and Technology, China; ${ }^{2}$ The Eighth Hospital of Wuhan, China

\subsection{6/gutjnl-2019-IDDFabstracts. 122}

Background Although several randomized controlled trials (RCTs) have shown that the addition of simethicone to polyethylene glycol (PEG) can improve bowel preparation, whether PEG plus simethicone had a beneficial role in the adenoma detection rate (ADR) during a colonoscopy has yet to be confirmed. The aim of this study was to clarify whether adding simethicone to a PEG solution could improve the effectiveness of the ADR.

Methods The PubMed, EMBASE, and Cochrane Library databases were searched for articles published prior to November 2018. The primary outcomes included the ADR and the polyp detection rate (PDR). The secondary endpoints included the efficacy of the colon preparation, procedure-related parameters, adverse events and willingness to repeat preparation. The odds ratios (ORs) and mean difference (MD) with 95\% confidence intervals (CIs) were pooled for discrete and continuous variables. The subgroup analyses and sensitivity analyses were performed.

Results Eighteen studies were eligible. Although there was no significant difference in the overall ADR (OR $=1.03$, 95\% CI: $0.89-1.18, \mathrm{P}=0.73$ ) between the groups with or without simethicone, a significant effect of simethicone for small adenomas $(<1 \mathrm{~cm})$ on the $\mathrm{ADR}$ was revealed $(\mathrm{OR}=2.36 ; 95 \% \mathrm{CI}$, $1.79-3.10 ; \mathrm{P}<0.00001)$. The subgroup analyses showed the beneficial effects of simethicone in the ADR among Asian populations $(\mathrm{OR}=1.45,95 \% \mathrm{CI}=1.12-1.87, \mathrm{P}=0.005)$ and

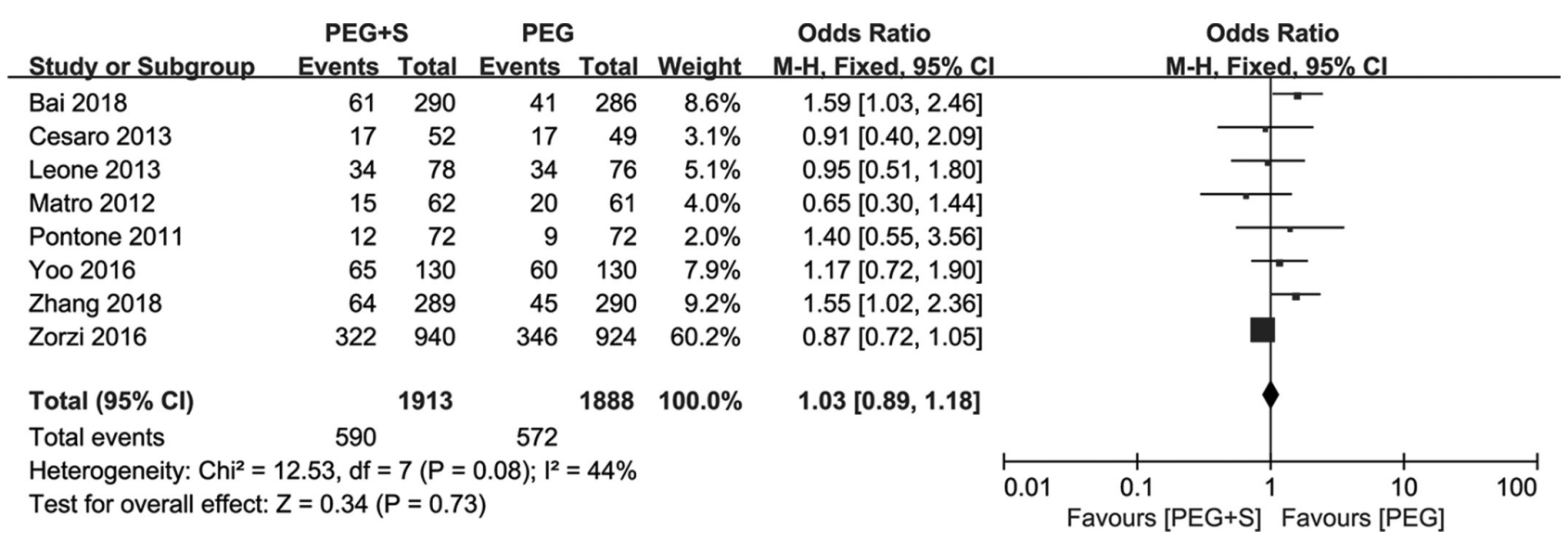

Abstract IDDF2019-ABS-0035 Figure 1 a) Forest plot of the effect of simethicone on overall ADR 